\title{
Motivations for Using Social Media: An Exploratory Factor Analysis
}

\author{
Jamal J. Al-Menayes ${ }^{1}$ \\ ${ }^{1}$ Department of Mass Communication, Kuwait University, Kuwait \\ Correspondence: Kuwait University, Department of Mass Communication, P.O. Box 23558, Safat, 13096, \\ Kuwait. E-mail: jamal@almenayes.com
}

Received: October 10, 2014

doi:10.5539/ijps.v7n1p43
Accepted: November 4, $2014 \quad$ Online Published: February 17, 2015

URL: http://dx.doi.org/10.5539/ijps.76n1p43

\begin{abstract}
This study aimed to examine motivations to use social media in a sample of university students. Grounded in the theory of Uses and Gratifications, the current research sough to delineate user motivations with exploratory factor analysis. To reach the study goals the researcher used cross-sectional survey methodology in which a questionnaire was distributed to 1327 undergraduate students with their consent. The analysis of the data revealed that almost all respondents used social media. Based on factor analysis results, their motivations for doing so are entertainment, information seeking, personal utility and convenience. These factors were positively related to the user's experience, time spent, and level of satisfaction with social media. Future studies should consider the cultural values of users and examine other possible motivations, taking into account their context.
\end{abstract}

Keywords: motivation, social media, uses and gratifications, factor analysis

\section{Introduction}

In spite of the much publicized criticism of the theoretical minimalism and methodological shortcomings ascribed to it, the uses and gratifications (U\&G) approach to media effects has proven its longevity and remarkable ability to attract new research and explorations over the past four decades. In many ways, U\&G provided the initial impetus to numerous empirical studies that brought us closer to the reasons and motivations behind people's utilization of mass media in all forms and modalities. According to Ruggiero (2000), adopting the uses and gratifications approach will allow researchers to outline and comprehend the significance of the uses of mediated communication, along with the numerous variables that should be taken into account for any type of media analysis. The current research examines the mitigating needs social media satisfy and lays down a typology of the various dimensions of their use. In addition, it will explore the primary concepts which can account for different reasons for their utilization.

First, I will discuss the history and recent standing of the Theory of uses and gratifications, exploring its theoretical underpinnings and at the various categorizations and organizations of uses and rewards covered in the research on this topic. Subsequently, I will explain the method used for the analysis, present the results of the data analysis, and, finally, my interpretation.

\section{Background of Uses and Gratifications}

With a foundation steeped in psychology and a distinct person-centric proclivity, the U\&G approach has a succinct overarching rule: people are different and they use mediated communication for a variety of purposes. As a result, the particular medium used has a specific aim and originate from factors at the individual and the social levels. These factors are characterized by the dynamic and purposive disposition of the participants, and operate in multiple contexts which mediate any type of communication behavior. Additionally, with the competitive nature of communication stimuli trying to satisfy the needs of participants; mediated communication has come to occupy a prominent role when pitted against face-to-face interaction.

While many researchers have identified themselves with the U\&G perspective, it nonetheless has been a topic of much scrutiny; either for providing an overly person-centered analysis (personal needs, reasons, behaviors) without much theoretical explication, or for using unconvincing research methods (e.g. the use of self-report data to reflect audience activity). Two more drawbacks come to mind: U\&Gs failure to account for media exposure, and its assertion that participants are cognizant of their inner-most motivations for their actions and are consistently able to explain them under any conditions (Sundar \& Limperos, 2013). 
Following the 1980s, there were attempts to overcome the evident psychological deficits of this approach by utilizing some of the precepts of semiology. As a result, communication behavior was examined not only with a view to its relationship to the media, but also in relation to the methods used by it to make sense of events based on media use. As summarized by Jensen and Jankowski (1993), new qualitative research point to audience ability to assign meanings to media content which in turn satisfy their needs and goals accordingly.

Recently, some research examined cyberspace using this approach utilizing a variety of classifications of motives for media consumption. These include Mukherji et al. (1998), (pastime, interpersonal utility, social exchanges, and scrutiny), Svennevig (2000), (entertainment, private relations, community, identity formation, observation, imagination, stimulation, and mood altering), Song et al. (2004), (virtual community), Krause et al. (2014), Mukherji et al. (1998), (personal identity, social relationships).

Other studies grounded in this theory paid attention to gratifications based on the communication medium used. For example Mosqueda and Garcia (2010) focused on chat rooms, and even though their study is clearly tentative and qualitative in nature, it nonetheless identified an elevation in sociability through coming in contact with known people or with unfamiliar persons, which enable new relationships to be formed along with a boost in buoyancy and self-assurance based on asserting one's own individuality and communication abilities. In this regard, it was evident that experience with gratifications was self-aware and directed. In a study of the social networking site MySpace, Fullwood, Sheehan and Nichollis (2009) conceptualized three primary drives for using the site: self-expression, establishing relations and personal impression management.

In another study, Park et al. (2009) found that university students joined Facebook to fulfill their need to socialize with their friends and for status conferral. Based on random Internet survey of university students in Texas $(n=2,603)$, the authors uncovered a significant correlation between the intensity of Facebook use and gratifications like feelings of personal fulfillment, self-assurance and taking part in public affairs. The socio-economic status of participants made no significant difference on gratifications obtained from using Facebook.

Continuing with the theme of social networks, research conducted by Urista et al. (2009) discovered two modalities of gratifications; social gratifications and communication gratifications. Social gratifications include things like the ability to keep in touch with family and friends, meeting like-minded individuals, social networking, maintaining relationships with old friends and being popular as indicated by the number of followers. Communication gratification, on the other hand, is found in the individual's capacity to control their communication, alongside the opportunity to establish new contacts on the site. Based on the findings of the research, social networking sites provide "selective, efficient, and immediate contact with others", which produces high levels of satisfaction. Moreover, they offer a flowing stream allowing the opportunity to get support from others. It also allows users to be content originators for new recipients in a habitual manner leading sometimes to addiction-like behavior.

Other studies found that, for younger individuals, social media are means for finding satisfaction and share their moods through a community culture from far away distances and with relative anonymity. Bloggers contribute to the emotional needs of others who may have difficulty expressing themselves in real life situations if the subject is too personal. In that regard, social media balance the lack of face-to-face contact for individuals who suffer from limited social resources (Quiroz, 2008).

Several efforts have been made to advance the ways in which uses and gratifications are classified to account for the complexity of the human experience. LaRose and Eastin (2002) proposed a social cognitive approach to determine people's expectations from cyberspace in the future in comparison to what they gained from their current and past experiences. This approach offered a number of dimensions along which to classify gratifications obtained. These include: novel sensory incentives, social gratifications, activity and personal incentives and status attainment. The researchers also explored the concept of self efficiency and self-regulation to predict Internet use.

Further studies in this vein have also look at conditions which can enhance the utilization of communication media at different life stages. Subrahamanyan et al. (2008) for example, studied how the members of a certain social group are different in their evaluation of the causes for use and the gratifications they attained from exploring the web. More specifically, the attention of these studies is focused on the receptiveness and capacity for assimilation of young people who have been called the net-generation, meaning those who did not know what life was like before the Internet (Prensky, 2011). Age and gender were found to be as pivotal factors playing a crucial role in what children and adolescents make of online media (Helsper, 2010). 
According to Uses and Gratifications theory, a match is expected between web surfing by adolescents and issues related to their growth, which includes identity formation and fashioning close associations with their cohorts (Subrahamanyam et al., 2008). In addition, a suggestion is made that adolescent development relies less on family, school and other institutions and more on peer groups over time (Giner et al., 1998). Livingstone (2010) has emphasized the significance adolescents ascribe to how well they project their identities and on the increasing part taken by social media such as Facebook or Twitter or Instagram in presenting the person's individuality as well as their relations with others.

\section{The Role of Motives in Uses and Gratifications Theory}

Audience activity is an essential part of the uses and gratifications perspective, and communication motivations are the main elements of audience activity (Rubin, 1993). Motives are general tendencies that affect people's behaviors taken to satisfy a need or desire. Motives are also a key concept in the study of online communications. Some have argued that online communications are mass media with the ability to satisfy interpersonal and mediated needs (Morris \& Ogan, 1996). The case is even stronger for social media which enable individuals to have real-time, interpersonal communication in mediated forms (Baek et al., 2011; Lee et al., 2012). Research on interpersonal motivations for using online media has delineated several taxonomies addressing the issue. For example, Flaherty et al. (1998), found that people utilized computers to satisfy: (a) interpersonal needs (e.g. inclusion, affection, control, relaxation, escape, and enjoyment); (b) needs normally satisfied by media (e.g. social interaction, passing time, habit, knowledge, and leisure); and (c) other needs (e.g. time shifting and making new friends), which are satisfied by social media.

\section{Research Questions}

Building on previous research, in which investigators measured motivations to communicate in a variety of ways in multiple contexts; my first research question addresses the motives behind utilizing social media. The second question asks about the correlates of the range of motivations people have for using social media. They are as follows:

RQ1: What are the motivations behind using social media?

RQ2: What are the correlates of various motivations for using social media?

\section{Method}

\subsection{Sample and Procedures}

A self-administered survey questionnaire was used for this study. Because young people constitute the core users of social media, the data were collected from a sample of purposively selected college students. College students enrolled in coursework in media studies at a large state university in Kuwait were asked to participate in this study. The questionnaires were distributed over a period of three months starting in March 2014. The total sample size was 1327. Arabic was the language used in the questionnaire.

Students were assured of anonymity and confidentiality, and participation was voluntary. The age of the participants ranged from 18 to 31 with $96 \%$ ranging between 18 to 25 years of age. The mean age of the participants in the study was 21.87 years. The participants were $395(29.8 \%)$ male and $931(70.2 \%)$ female. This gender distribution reflects the enrollment profile of the university student body which is $70 \%$ female. Finally, since this is a state university, the overwhelming majority of students are Kuwaiti nationals by law so there was no need to record nationality. The self-administered questionnaires were distributed during regularly scheduled class sessions. The instrument consisted of both Likert scale questions used to measure the individual's perceptions, attitudes and behaviors as well as demographic questions and questions about media use patterns.

\subsection{Measurement of Motives for Using Social Media}

The study utilized a combination of interpersonal, media, and new technology motives to measure motives for using social media. To construct the social media motives scale, the study combined interpersonal (affection, inclusion, and control), media (entertainment, habit, information, social interaction, escape, surveillance, pass time, and relaxation), and social media (time control, convenience, economy, and expressive need) motives. Three items were used to represent 15 possible a priori categories, and adapted several statements from previous research to the social media context. Respondents were asked how much their reasons for using social media correspond with the statements in the questionnaire on a 5-point Likert scale (5=exactly, 1=not at all).

The motivation items were subjected to principal-component analysis with varimax rotation to extract and interpret possible social media motives factors. An eigenvalue of 1.0 or greater was required to retain a factor, which also had to contain two items loading criterion at a minimum. The retained factors were transformed into 
numeric variables and saved in the data file for subsequent analysis. The factor results accounted for $56.5 \%$ of the variance.

\section{Experience with Social Media}

Respondents were asked a single question about the time they first started using social media. The response set ranged between (1) less than a year to (8) more than six years.

\section{Hours Spent Using Social Media per Day}

Respondents were asked a single question about the total number of hours spent using social media daily on an eight point scale: (1) less than two hours, (2) from two to 4 hours, (3) from 4 to 6 hours, (4) from 6 to eight hours, (5) from eight to 10 hours, (6) from 10 to 12 hours, (7) from 12 to 14 hours, (8) more than 14 hours.

\section{Satisfaction with social media}

Similar to Palmgreen and Rayburn (1985), the study used a single-item to measure satisfaction with social media use. Respondents were asked to indicate: "Overall, how satisfied are you with social media in what it does in providing you with the things you are seeking?" Response options ranged from extremely satisfied (5) to not at al satisfied (1). This measure had a mean of $4.00(S D=0.79)$.

\section{Results}

\subsection{Descriptive Statistics of Social Media Use Patterns}

Before discussing the data analysis, I will first present some key descriptive statistics of the sample relevant to the research questions. Table 1 contains answers to questions about whether or not respondents use social media at all, whether they use social media while driving and if they met anyone in person through social media. Ninety nine percent of the sample reported they use social media, $51 \%$ said they use it while driving, and $58 \%$ mentioned they met someone in person they first ran into on social media.

Table 2 ranks the respondents favorite social media apps. Whatsapp is by far the favorite $(50 \%)$ followed by Instagram (23\%), and lastly Twitter (16\%)

Table 1. Descriptive summary of social media use patterns

\begin{tabular}{lll}
\hline Variable & Yes (\%) & No (\%) \\
\hline Do you use social media (SM)? & $1316(99)$ & $10(0.8)$ \\
Do you use SM while driving? & $648(51)$ & $676(49)$ \\
Have you met anyone in person through SM? & $771(58)$ & $555(42)$ \\
\hline
\end{tabular}

$n=1326$

Table 2. Popularity of social media applications

\begin{tabular}{ll}
\hline Which social medium do you use the most? & $n(\%)$ \\
\hline Whatsapp & $663(50)$ \\
Instagram & $302(23)$ \\
Twitter & $216(16)$ \\
Others & $111(8)$ \\
\hline
\end{tabular}

Valid $n=1293$

\subsection{Motives for Using Social Media}

Twenty two likert-scale items were used in the questionnaire to estimate the dimensions underlying our key variable "motives for using social media". Exploratory factor analysis with varimax rotation was performed on these items to ascertain their underlying factors. As a result of the analysis four items were discarded due to eigenvalues under 1 . All items were standardized to ensure they were on equal footing. 
Table 3 shows the results of this analysis. As we can see, there are five factors representing the underlying dimensions of motivation behind the utilization of social media. They are: entertainment, personal utility, information seeking, convenience and altruism. Below, I will discuss each of these factors in more detail.

Table 3. Factor analysis of gratifications obtained from social media

\begin{tabular}{|c|c|c|c|c|c|c|c|}
\hline Factors & Mean & $S D$ & 1 & 2 & 3 & 4 & 5 \\
\hline \multicolumn{8}{|l|}{ Entertainment } \\
\hline 1. I use it to kill time. & 4.41 & 0.86 & 0.784 & & & & \\
\hline 2. When I have nothing else to do. & 4.29 & 0.97 & 0.755 & & & & \\
\hline 3. To occupy my time. & 4.29 & 0.90 & 0.805 & & & & \\
\hline 4. Because it entertains me. & 4.23 & 0.84 & 0.732 & & & & \\
\hline 5. Because I enjoy using it. & 4.31 & 0.78 & 0.735 & & & & \\
\hline \multicolumn{8}{|l|}{ Personal utility } \\
\hline 6. To join conversations. & 3.70 & 1.06 & & 0.603 & & & \\
\hline 7. To join groups. & 2.99 & 1.24 & & 0.604 & & & \\
\hline 8. I enjoy answering questions. & 3.07 & 1.22 & & 0.618 & & & \\
\hline 9. To listen to other's opinion. & 3.66 & 1.18 & & 0.692 & & & \\
\hline \multicolumn{8}{|l|}{ Information seeking } \\
\hline 10. For research and homework. & 3.65 & 1.09 & & & 0.599 & & \\
\hline 11. To get free information. & 4.03 & 0.99 & & & 0.807 & & \\
\hline 12. To search for information. & 4.11 & 0.95 & & & 0.829 & & \\
\hline 13. To know what's going on. & 4.25 & 0.87 & & & 0.528 & & \\
\hline \multicolumn{8}{|l|}{ Convenience } \\
\hline 14. Because it's free. & 3.55 & 1.27 & & & & 0.501 & \\
\hline 15. It's easier than meeting. & 3.61 & 1.31 & & & & 0.794 & \\
\hline 16. Others can answer anytime. & 3.47 & 1.27 & & & & 0.799 & \\
\hline \multicolumn{8}{|l|}{ Altruism } \\
\hline 17. To help others. & 3.12 & 1.04 & & & & & 0.680 \\
\hline 18. To encourage others do work. & 3.10 & 1.15 & & & & & 0.592 \\
\hline Eigenvalue & & & 5.61 & 2.71 & 1.65 & 1.36 & 1.07 \\
\hline$\%$ of variance explained & & & 25.51 & 12.34 & 7.53 & 6.21 & 4.90 \\
\hline Cronbach's alpha & & & 0.84 & 0.72 & 0.73 & .610 & 0.65 \\
\hline
\end{tabular}

Note. Loadings $<0.50$ were suppressed.

The first factor, entertainment, accounted for $25.61 \%$ of the variance after rotation. It contained 5 items from a priori categories reflecting this dimension (Cronbach $\alpha=.84$ ). It was the only factor containing statements primarily from entertainment oriented categories. Personal utility consisted of four items, all of which comprised an a priori category (Cronbach $\alpha=.72$ ). They are "to join conversations", "to join groups", "to answer questions", and "to listen to others." It accounted for $12.34 \%$ of the variance. "Information seeking" also contained four items: "research", "getting free information", "searching for information", and "to know what's going on" categories (Cronbach $\alpha=.73$ ). This factor explained $7.53 \%$ of the variance. "Convenience" included three items representing the underlying dimension. They are "it's free", it's easier", and "others can answer anytime" (Cronbach $\alpha=.61$ ). It explained $6.21 \%$ of the variance. Finally, altruism contained two items "to help others" and "to encourage others" (Cronbach $\alpha=.65$ ). It explained $4.90 \%$ of the variance after rotation. 
Entertainment $(M=4.30, S D=.87)$ and information seeking $(M=4.01, S D=.97)$ had the highest mean scores. None of the other factors were as salient as the previous two. Primarily, social media users in this sample regarded the medium as a convenient source of entertainment and information. There were no significant differences between males and females on these factors with the exception of two. Regression analysis revealed that females were more like than males to use social media for entertainment and they were less likely than males to use it for personal utility $(\beta=.179, p<.001 ; \beta=-.078, p<.001$, respectively).

\subsection{Correlates of Motivations for Using Social Media}

RQ2 asked about the variables correlated with motivations for using social media. Table 3 shows the results of the statistical analysis used to examine these relationships. Pearson product moment coefficient was calculated for three scale variables included in the survey, the ones that are believed to be of interesting theoretical possibilities. These variables are "the amount of time since they first started using social media", "time spent using social media daily", and "satisfaction with the functionalities social media provide." All variables were measured using quantitative scales ranging from 1 (the least score) to 8 (the highest possible score).

Table 4. Pearson correlation between motivation factors and social media variables

\begin{tabular}{llll}
\cline { 2 - 4 } Variables & Start & Time & Satisfaction \\
\cline { 2 - 4 } Entertainment & $.16^{* *}$ & $.29^{* *}$ & $.23^{* *}$ \\
Personal utility & $.10^{* *}$ & $.15^{* *}$ & $.17^{* *}$ \\
Information seeking & .02 & $-.06^{*}$ & $.09^{* *}$ \\
Convenience & -.00 & $.08^{* *}$ & $.12^{* *}$ \\
Altruism & .00 & -.01 & .05 \\
$*_{p}^{*}<.05 .{ }^{* *} p<.01^{*}$ & & &
\end{tabular}

Table 4 displays the results of this analysis. As we can see, the more experience a respondent has with social media the more likely he or she will utilize it for entertainment and personal utility $(\mathrm{r}=.16, \mathrm{r}=.10, \mathrm{p}<.01$, respectively). Time spent using social media on a daily basis is positively correlated with all dimensions with the exception of information seeking where it is negatively related $(r=-.06, p<.05)$. This last finding indicates that users who spent a lot of time using social media are less likely to use it for information seeking and more likely to use it for entertainment, personal utility and convenience.

Finally, and not surprisingly, satisfaction with the functionality of social media is positively correlated with all motivation dimensions. This means individuals who are satisfied with what social media have to offer are equally driven by entertainment, personal utility, information seeking and convenience.

\section{Conclusion}

This study followed the uses and gratifications approach to mass media effects. The central hypothesis of which is the effects of mediated communication are contingent upon the participant's needs and motivations. Taking this theory into consideration will help us to frame and understand the reasons people utilize media the way they do. To satisfy this condition, the current research looked deep into the social and personal needs that social media satisfy in all of its modalities. Furthermore, an effort has been made to lay down a typology of the various dimensions of motivations using empirical research. Based on factor analysis of responses from a sample of 1326 students, five dimensions emerged: entertainment, personal utility, information seeking, convenience and altruism. These dimensions were subsequently correlated with communication related variable to measure their impact. Results showed that the longer the user's experience with social media, the more he or she will use it for entertainment and personal utility. The remaining variables: "time spent using social media per day" and "satisfaction with social media" were both correlated with all motivation dimensions except altruism which had no significant correlation with any dimension. A useful path for further research would take into account the cultural values and their impact on the relationship between motivation and its correlates. Future studies should also continue to examine other possible motivation factors through more qualitative methods such as focus groups and participant observation. This methodological approach known as "triangulation" will help increase the depth and complexity of the variables used in the future. It will also enable us to take into account any future trends given that we are dealing with a phenomenon populated largely by youth. 


\section{Limitations}

There are a few limitations that might affect the generalizability of the findings. First, the cross-sectional data utilized for this study do not merit an assertion of any causal relationships between the independent and dependent variables. furthermore, the sample which had female to male ratio of 2:1 could skew the results by showing more variance in the former compared to the latter. Perhaps a quota sample with equal numbers of males and females should have been used to insure that we don't get gender differences because of the uneven distribution.

Second, the definition of some constructs might limit the scope of the study. The main independent variables were based on self-reports. For example, the variable "time spent using social media" was measured by asking participants how much time they spend using social media on a typical day. Although this question measures usage time accurately, some doubt remains as to whether users are active all the time they are logged on to a specific application. Heavy and light users can be better analyzed in future studies by asking how many messages are sent or received each day.

Third, the definition of typical social media use provided a viable empirical description to examine the research questions, but it might not precisely reflect the complexity of an individual's use patterns. It is possible that each individual uses several social media functions (e.g. Chat, post pictures, audio or video) each day. Researchers would benefit from developing tools for capturing the complexity of social media and user patterns.

Finally, the fact that data collected for this study of social media use was limited to college students should be taken into consideration. Investigating only college students' social media usage might not wholly explain the electronic social networking behavior in general. Future researchers are also strongly encouraged to attempt to replicate these results by analyzing users of different social media platforms (e.g. Twitter, Instagram...etc.) separately to account for the various features they offer.

\section{Acknowledgement}

This research was supported and funded by Kuwait University Research Grant No. (AM01/14)

\section{References}

Baek, K., Holton, A., Harp, D., \& Yaschur, C. (2011). The links that bind: Uncovering novel motivations for linking on Facebook. Computers in Human Behavior, 27, 2243-2248. http://dx.doi.org/10.1016/j.chb.2011.07.003

Flaherty, L. M., Pearce, K. J., \& Rubin, R. B. (1998). Internet and face-to-face communication: Not functional alternatives. Communication Quarterly, 46, 250-268. http://dx.doi.org/10.1080/01463379809370100

Fullwood, G., Sheehan, N., \& Nigholls, W. (2009). Blog function revisited: A content analysis of MySpace. Cyber psychology \& Behavior, 12(6), 685-689. http://dx.doi.org/10.1089/cpb.2009.0138

Giner, Salvador, Lamo de Espinosa, Emilio y Torres, \& Gristóbal. (1998). Dicdonario de Sociohgía. Alianza, Madrid.

Helsper, E. J. (2010). Gendered Internet use across generations and life stages. Communication research, 37(3), 503-520.

Jensen, K. B., \& Jankowski, N. W. (1993). Qualitative methodologies for mass communication research. Routledge: London.

Krause, A. E., North, A. C., \& Heritage B. (2014). The uses and gratifications of using Facebook music listening applications. Computers in Human Behavior, 39, 71-77. http://dx.doi.org/10.1016/j.chb.2014.07.001

Larose, R., \& Eastin, M. S. (2002). A social cognitive explanation of Internet? In Uses and gratifications: Toward a new theory of media attendance, .International Communication Association.

Lee, J., Lee, M., \& Choi, H. (2012). Social network games uncovered: Motivations and their attitudinal and behavioral outcomes. Cyberpsychology, Behavior, \& Social Networking, 15(12), 643-648. http://dx.doi.org/10.1089/cyber.2012.0093

Livingstone, S. (2010). Taking risky opportunities in youthful content creation: Teenagers' use of social networking sites for intimacy, privacy and self-expression. New Media \&. Society, 10(3), 393-411.

Morris, M., \& Ogan, C. (1996). The Internet as mass medium. Journal of Communication, 46(1), 39-50.

Mosqueda, R. P., \& G. Martinez (2010), Rasgos de personalidad y gratificaciones de jóvenes usuarios del chat. Razón y Palabra, 72. 
Mukherji, J., Mukherji, A., \& Nicovich, S. (1998). Understand in dependency and use of the Internet: A Uses and Gratifications Pers. Paper presented at the American Marketing Association, Boston, August. pective.

Palmgreen, P., \& Rayburn, J. D. (1985). A comparison of gratification models of media satisfaction. Communication monographs, 52, 334-346.

Park, N. K., \& Valenzuela, K. S. (2009). Being immersed in social networking environment: Facebook groups, uses and gratifications, and social outcomes. Cyberpsychology and behavior, 12(6), 729-733. http://dx.doi.org/10.1089/cpb.2009.0003

Prensky, M. (2011). Digital natives, digital immigrants. On the Horizon, 9(5), 1-6.

Quiroz, M. T. (2008). La edad de la pantalla. Tecnobgias interactivas y jóvenes peruanos. Fondo Editorial de la Universidad de Lima, Lima.

Rubin, A. M. (1994). Media uses and effects: A uses-and-gratifications perspective. In J. Bryant, \& D. Zillman (Eds.), Media Effects: Advances in Theory and Research, (pp. 417-436). Hillsdale, NJ: Erlbaum.

Ruggiero, T. E. (2000). Uses and Gratifications theory in the $21^{\text {st }}$ century. Mass Communication and Society, $3(1), 3-37$.

Song et al. (2004). Internet gratifications and Internet addiction: On the uses and abuses of new media, Cyberpsychology \& Behavior, 7(4), 384-394. http://dx.doi.org/10.1089/cpb.2004.7.384

Subrahamanyam et al. (2008). Online and offline social networks: Use of social networking sites by emerging adults. Journal of Applied Developmental Psychology, 29, 420-433. http://dx.doi.org/10.1016/j.appdev.2008.07.003

Sundar, S. S., \& Limperos, A. (2013). Uses and Grats 2.0: New gratifications for new media. Journal of Broadcasting and Electronic Media, 57(4), 504-535. http://dx.doi.org/10.1080/08838151.2013.845827

Svennevig, M. (2000). Needs, not needs: Researching technological change. International Journal of Advertising, 9, 654-663.

Urista, M. A., Dong, Q., \& Day, K. (2009). Explaining why young adults use Myspace and Facebook through uses and gratifications theory. Human Communication, 12(2), 215-229.

\section{Copyrights}

Copyright for this article is retained by the author(s), with first publication rights granted to the journal.

This is an open-access article distributed under the terms and conditions of the Creative Commons Attribution license (http://creativecommons.org/licenses/by/3.0/). 